\title{
Quasifission Dynamics in the Formation of Superheavy Elements
}

\author{
D.J. Hinde ${ }^{1, \star}$, M. Dasgupta ${ }^{1}$, D.Y. Jeung ${ }^{1}$, G. Mohanto ${ }^{1, \star \star}$, E. Prasad ${ }^{1, \star \star \star}$, C. Simenel ${ }^{1}$, E. Williams ${ }^{1}$, I.P. Carter ${ }^{1}$, \\ K.J. Cook ${ }^{1}$, Sunil Kalkal ${ }^{1}$, D.C. Rafferty ${ }^{1}$, E.C. Simpson ${ }^{1}$, H.M. David ${ }^{2}$, Ch.E. Düllmann ${ }^{2,3,4}$, and J. Khuyagbaatar ${ }^{2,3}$ \\ ${ }^{1}$ Department of Nuclear Physics, Research School of Physics and Engineering, \\ Australian National University, Canberra, ACT 2601, Australia \\ ${ }^{2}$ GSI Helmholtzzentrum für Schwerionenforschung, D-64291 Darmstadt, Germany \\ ${ }^{3} \mathrm{Helmholtz} \mathrm{Institute} \mathrm{Mainz,} \mathrm{D-55099} \mathrm{Mainz,} \mathrm{Germany}$ \\ ${ }^{4}$ Johannes Gutenberg Universität Mainz, D-55099 Mainz, Germany
}

\begin{abstract}
Superheavy elements are created through the fusion of two heavy nuclei. The large Coulomb energy that makes superheavy elements unstable also makes fusion forming a compact compound nucleus very unlikely. Instead, after sticking together for a short time, the two nuclei usually come apart, in a process called quasifission. Mass-angle distributions give the most direct information on the characteristics and time scales of quasifission. A systematic study of carefully chosen mass-angle distributions has provided information on the global trends of quasifission. Large deviations from these systematics at beam energies near the capture barrier reveal the major role played by the nuclear structure of the two colliding nuclei in determining the reaction outcome, and thus implicitly in hindering or favouring superheavy element synthesis.
\end{abstract}

\section{Introduction}

Superheavy elements (SHE) are formed by heavy-ion fusion reactions. Fusion cross sections can be considerably suppressed [1] by quasifission [2]. This non-equilibrium process results when the combined di-nuclear system, formed as the two nuclear surfaces stick together, subsequently separates into two (fission-like) fragments, with the initial kinetic energy largely or completely damped. Quasifission can occur very rapidly, typically in less than $10^{-20} \mathrm{~s}$, before a compact compound nucleus can be reached [2-5]. The probability of quasifission $\left(\mathrm{P}_{Q F}\right)$ can be very large, thus the complementary probability of compound nucleus formation $\left(\mathrm{P}_{C N}=1-\mathrm{P}_{Q F}\right)$ can be small, quite likely lower than $10^{-3}$ in reactions forming superheavy elements. Understanding the competition between quasifission and fusion is thus very important in predicting the optimal fusion reactions to use to form new elements and isotopes in the superheavy mass region.

A key characteristic, important for superheavy element formation, is the "sticking time" following contact of the two nuclear surfaces [6]. It is expected that the sticking time is correlated with $\mathrm{P}_{C N}$ : where the sticking time is longer, then $\mathrm{P}_{C N}$ would be expected to be larger (more favourable for SHE synthesis). The average sticking time can be extracted from measurements of quasifission angular distributions. The two colliding nuclei always ap-

\footnotetext{
${ }^{\star}$ e-mail: david.hinde@anu.edu.au

$\star \star$ Current address: BARC, Mumbai, India

${ }^{\star \star}$ Permanent address: Department of Physics, School of Mathematical and Physical Sciences, Central University of Kerala, Kasaragod 671314, India.
}

proach each other along the beam axis, and after contact rotate with angular velocities that can be calculated. Measurement of the rotation angle thus allows estimation of the sticking time. As the system rotates, mass flow also occurs between the two nuclei. Measurement of the velocity vectors of both fragments gives direct information on the centre-of-mass angle and mass-ratio of the fragments at scission, defined as $M_{R}=M_{1} /\left(M_{1}+M_{2}\right)$, as well as providing excellent discrimination against fission events resulting from peripheral (transfer-induced) processes $[4,7]$. The mass-ratio (or mass) plotted as a function of the centre-of-mass angle is referred to as a massangle distribution, or MAD. This gives direct information on the dynamical time scales, as long as the system undergoes less than a full rotation (taking $\sim 10^{-20} \mathrm{~s}$ ). This is usually the case for collisions of heavy nuclei, as shown first by measurements at GSI [2, 8], and by later results from ANU [3-5, 9-15]. Inter-relationships of different measurements, and background to the results presented here are given in recent conference proceedings [16-20].

\section{Experimental Setup}

The detector configuration used at the Australian National University (ANU) to measure MAD is shown in Fig.1. It consists of two or three large area multi-wire proportional counters (MWPC). They detect reaction products from the interaction of the pulsed beam with targets typically 50$200 \mu \mathrm{g} / \mathrm{cm}^{2}$ in thickness, oriented with the normal to the target face at $60^{\circ}$ to the beam. This eliminates shadowing of the detectors by the target frame. The average en- 


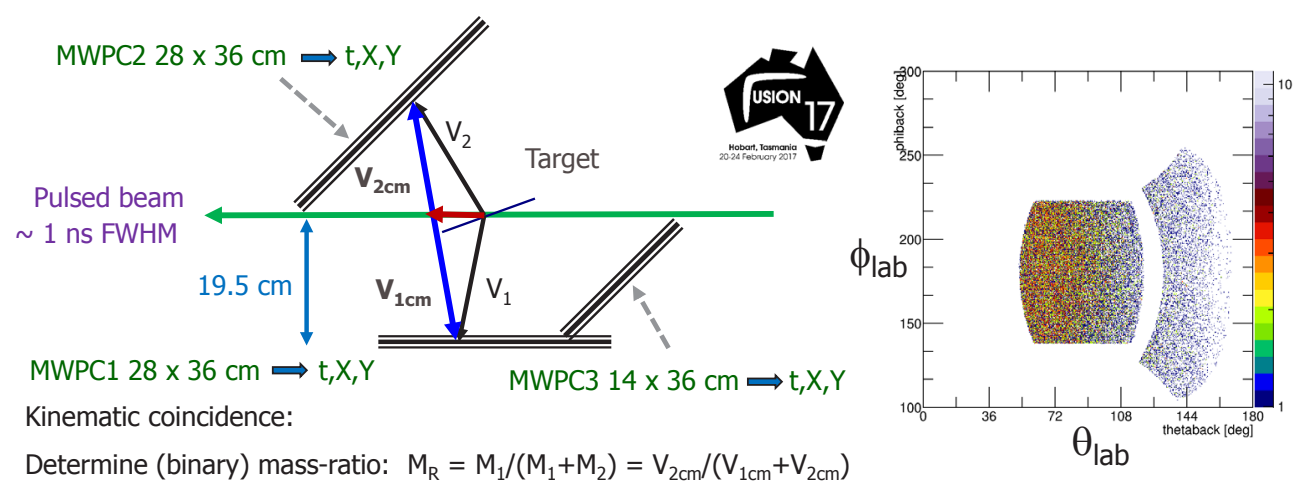

Figure 1. Enhanced ANU experimental setup to measure fission mass-angle distributions over the widest achievable angular range. The angular coverage of the MWPC1 and MWPC 3 detectors, for events in coincidence with MWPC2, are shown in scattering angle $\theta$ and azimuthal angle $\phi$.

ergy loss of the beam and the fragments in the target material is corrected for in the analysis. The wide coverage of MWPC1 and MWPC3 in scattering angle $(\theta)$ and azimuthal angle $(\phi)$ for coincidence events with MWPC2 is demonstrated in Fig.1. From the good resolution of the detectors in position $(\sim 1 \mathrm{~mm})$ and time $(<500 \mathrm{ps})$, and knowledge of the interaction time of the beam pulse with the target, the velocity vectors of the detected particles are determined. From the deduced velocity vectors in the centre-of-mass frame, for each binary event the centre-ofmass angle and the mass-ratio are determined, thus generating the MAD.

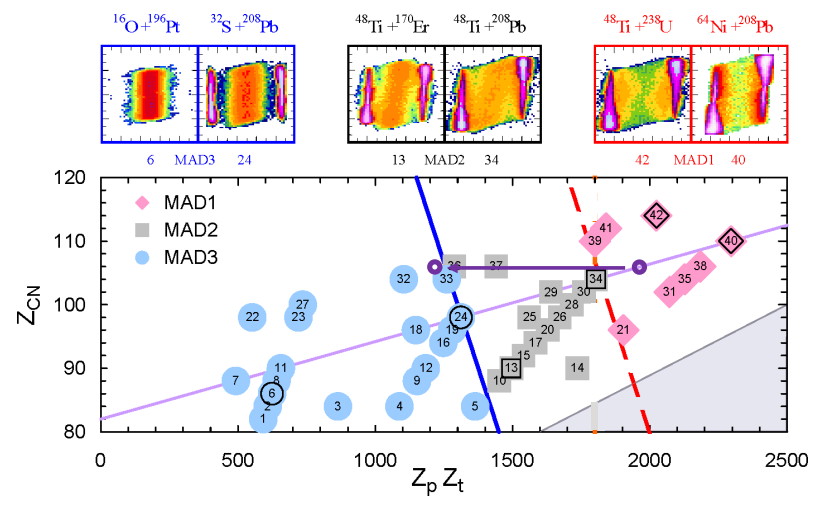

Figure 2. The symbol indicates the classification of MAD observed, shown as a function of the charge product of the colliding nuclei $\mathrm{Z}_{p} \mathrm{Z}_{t}$ and the atomic number of the compound nucleus $\mathrm{Z}_{C N}=\mathrm{Z}_{p}+\mathrm{Z}_{t}$. The numbers refer to the specific reaction in Ref.[4]. The diagonal full blue line represents the empirical boundary between reactions with no mass-angle correlation (left) and those that have (right). The diagonal red dashed line indicates the boundary of reactions which no longer exhibit a peak at symmetry in the angle-integrated fission mass distribution. The thin purple line represents the locus of reactions with $\mathrm{Pb}$. Examples of each type of MAD are shown in the panels above, with their reaction number. The purple circles and arrow refer to $\mathrm{Cr}+\mathrm{Pb}$ measurements discussed towards the end of the paper.

\section{MAD systematics}

Experimental MAD have been divided into three categories [4] having: (i) a mass-angle correlation with a minimum yield at mass-symmetry - associated with short sticking times (MAD1); (ii) a mass-angle correlation with peak yield at mass-symmetry - resulting from intermediate sticking times (MAD2); and (iii) no significant massangle correlation and a narrow mass-distribution - associated with long sticking times, including fission following fusion (MAD3). Examples of each type of MAD are shown in the upper panels of Fig.2. The systematic trends of MAD characteristics with the identity of the two colliding nuclei was studied [duRietz13], to determine global trends of quasifission dynamics. This is in analogy with the evaluation of the smooth liquid drop model dependence of nuclear masses on $\mathrm{N}$ and $\mathrm{Z}$, where deviations highlight the effects of nuclear structure. Choosing bombarding energies $\mathrm{E}$ well-above the mean capture barrier $\mathrm{B}$ (around $\mathrm{E} / \mathrm{B}=1.08$ ), nuclear structure effects were minimised. It was found [duRietz13] at these bombarding energies that the MADs are indeed strongly correlated with global variables. The simplest variables are the Coulomb repulsion in the entrance channel (related to the product of the proton numbers of the projectile and target nuclei $\mathrm{Z}_{p} \mathrm{Z}_{t}$ ), and the compound nucleus atomic number $\mathrm{Z}_{C N}$, as illustrated in Fig.2. However, for particular cases, it has been found that the nuclear structure of the nuclei in the entrance channel is extremely important in determining the sticking times and MAD characteristics. This relates to doubly-magic neutron-rich nuclei such as ${ }^{48} \mathrm{Ca}$ and ${ }^{208} \mathrm{~Pb}$, and prolate deformed actinide nuclei, all used in SHE formation reactions, as described below.

\section{Effect of spherical closed shells}

To investigate in detail the effect of closed shells in the entrance channel on quasifission probabilities and characteristics, measurements [13] of MADs were made for ${ }^{40,44,48} \mathrm{Ca}$ projectiles bombarding targets of ${ }^{208,204} \mathrm{~Pb}$ (forming ${ }^{248,252,256} \mathrm{No}$ with $\mathrm{Z}_{C N}=102$ ), and for ${ }^{48} \mathrm{Ti}$ bombarding ${ }^{200} \mathrm{Hg}\left({ }^{248} \mathrm{No}\right.$ ) and ${ }^{208} \mathrm{~Pb}$ (forming ${ }^{256} \mathrm{Db}$ with $\mathrm{Z}_{\mathrm{CN}}$ 


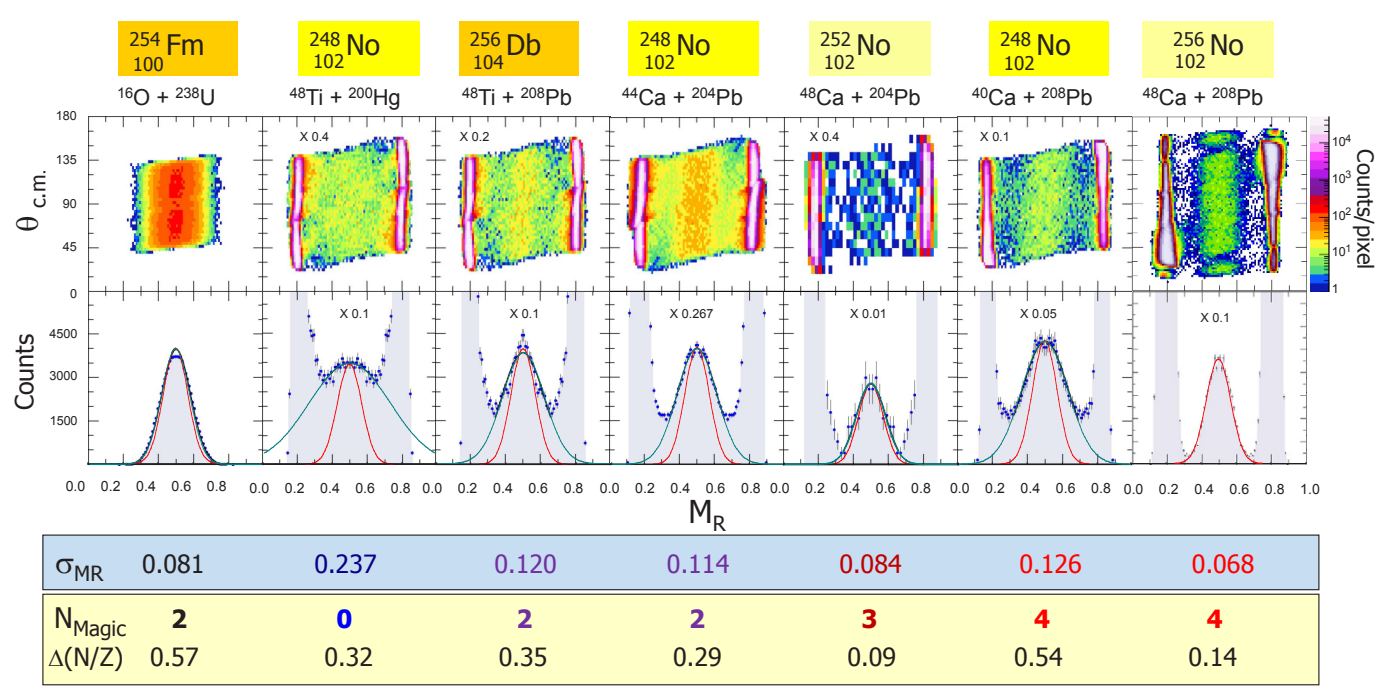

Figure 3. Measured mass-angle distributions for the indicated reactions at $\mathrm{E} / \mathrm{B} \sim 0.98$ (upper panels), compared to ${ }^{16} \mathrm{O}+{ }^{238} \mathrm{U}$ (left) measured at an above-barrier energy, where fast quasifission would be expected to be negligible. The MAD for ${ }^{48} \mathrm{Ca}+{ }^{208} \mathrm{~Pb}$ shows a dip in coverage at $30^{\circ}$ and $150^{\circ}$ due to the gap between detectors shown in Fig.1. In the projected mass ratio spectra for $45^{\circ}<\theta_{c . m}$. $<135^{\circ}$ (lower panels) the scale factor multiplies the counts scale on the left. Gaussian functions with $\sigma_{M R}=0.07$ (thin red lines) are shown for reference. Gaussian fits to the region around $\mathrm{M}_{R}=0.5$ are shown (turquoise lines), whose standard deviations $\sigma_{M R}$ are tabulated, along with the total number of magic numbers of the projectile and target nuclei $\mathrm{N}_{\text {Magic }}$, and the difference $\Delta(\mathrm{N} / \mathrm{Z})$ between the projectile and target nucleus N/Z ratios. (Adapted from Ref.[13])

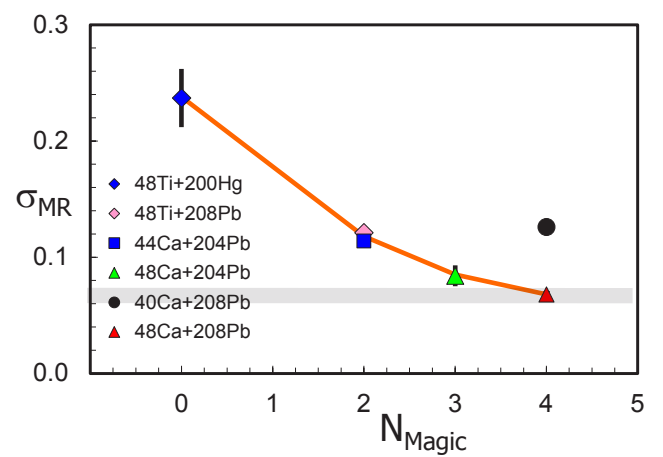

Figure 4. Fitted standard deviations $\sigma_{M R}$ of the fission-like mass distributions as a function of the number $N_{m}$ of magic numbers in the entrance channel, for the reactions shown in Fig.3. The line guides the eye. The expectations of $\sigma_{M R}$ for fusion-fission are in the range 0.06 to 0.08 . The value of $\sigma_{M R}$ for the ${ }^{40} \mathrm{Ca}+{ }^{208} \mathrm{~Pb}$ reaction is large, and the MAD in Fig. 3 shows a mass-angle correlation. This was attributed [13] to the N/Z mismatch between the projectile and target nuclei causing transfer reactions early in the collision, which attenuate the effect of the four magic numbers in the entrance channel.

=104). Measurements were made a few percent below the average fusion barrier energy to give "gentle" collisions with minimal relative velocity at contact. The MAD and projected $\mathrm{M}_{R}$ spectra are shown in Fig.3, together with reference data for the ${ }^{16} \mathrm{O}+{ }^{238} \mathrm{U}$ reaction at an above-barrier energy, forming ${ }^{254} \mathrm{Fm}$. The standard deviations $\sigma_{M R}$ of the Gaussian fits are tabulated.

Despite having similar or identical $\mathrm{Z}_{p} \mathrm{Z}_{t}$, and forming similar or identical compound nuclei, values of $\sigma_{M R}$ differ by more than a factor of three, indicating a significant vari- ation in the characteristics and probability of quasifission. Indeed, for the most recent (unpublished) MAD data for the ${ }^{48} \mathrm{Ca}+{ }^{208} \mathrm{~Pb}$ reaction, the characteristics appear consistent with a fusion-fission mechanism. To reveal the systematic behaviour, the variation of $\sigma_{M R}$ with the total number of magic numbers $\mathrm{N}_{\text {Magic }}$ in the entrance channel [13] is shown in Fig.4. Large values of $\sigma_{M R}$ are found for no magic numbers, reducing to values expected for fusionfission for maximal $\mathrm{N}_{\text {Magic }}$ (for ${ }^{48} \mathrm{Ca}+{ }^{208} \mathrm{~Pb}$ ). This suggests that reactions involving nuclei having several magic numbers form a compact compound nucleus with higher probability. It seems likely that this is associated with reduced energy dissipation as the two nuclei interpenetrate, allowing more compact shapes to be reached. The exception is the reaction ${ }^{40} \mathrm{Ca}+{ }^{208} \mathrm{~Pb}$, with 4 magic numbers, where the mass-width and MAD are consistent with quasifission being much more likely. It was suggested [13] that transfer reactions before contact can be expected to attenuate the entrance-channel magicity in this reaction, driven by the extreme mismatch of the $\mathrm{N} / \mathrm{Z}$ ratios of projectile and target nuclei. This was supported by TDHF calculations [13]. Supporting the "magic number" hypothesis, systematic analysis of heavy element cross sections in many reactions forming Th isotopes indicated somewhat enhanced fusion probabilities with two magic numbers in the entrance channel [21].

More recent ANU quasifission results, for the reaction of isotopes of $\mathrm{Cr}$ with $\mathrm{Pb}$, support these conclusions regarding magicity and N/Z matching. MAD and massratio projections at a sub-barrier energy for each reaction studied are shown in Fig.5. The three reactions on the left all form the same compound nucleus ${ }^{258} \mathrm{Sg}$. The panels are ordered from left to right first by the num- 


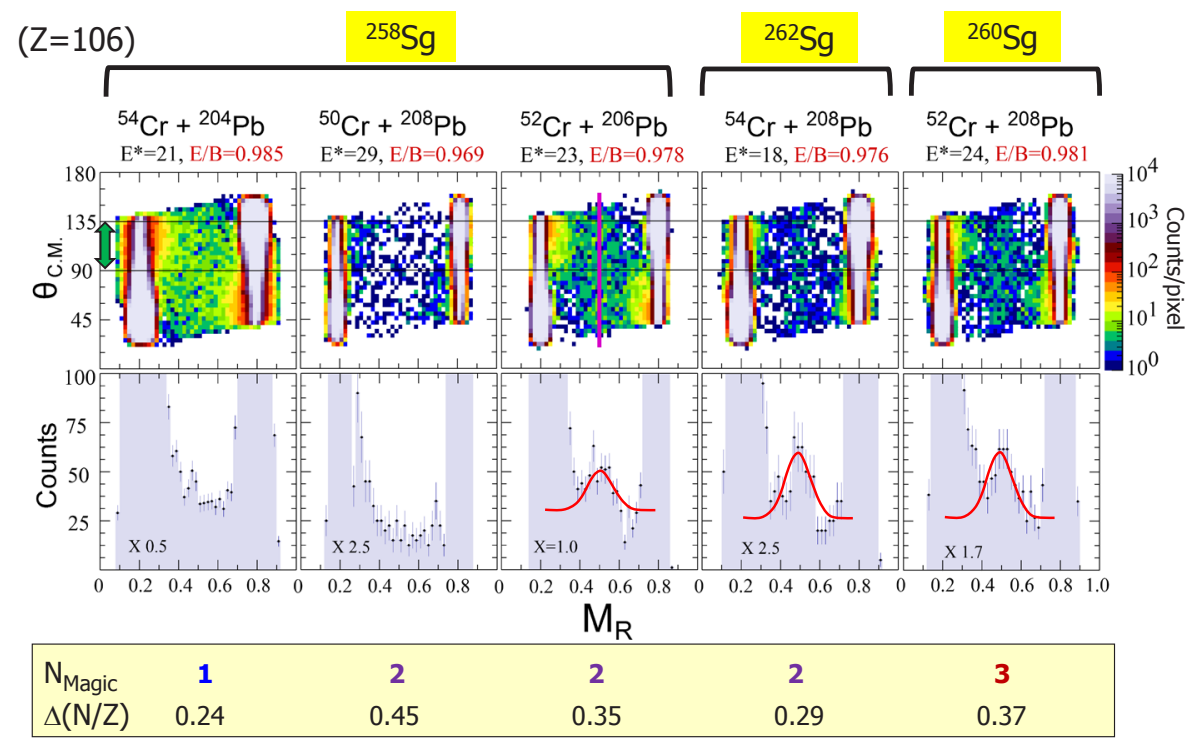

Figure 5. MAD and projected mass-ratio distributions for backward angles from $90^{\circ}$ to $135^{\circ}$ (as indicated in the MAD plots), for reactions of ${ }^{50,52,54} \mathrm{Cr}$ isotopes with ${ }^{204,206,208} \mathrm{~Pb}$. Sub-barrier beam energies (denoted by E/B), resulted in the low excitation energies $\mathrm{E}^{*}$. As in Fig.4, the total number of magic numbers of the projectile and target nuclei $\mathrm{N}_{\text {Magic }}$, and the difference $\Delta(\mathrm{N} / \mathrm{Z})$ between the projectile and target nucleus N/Z ratios are shown for each reaction. The reaction outcome changes from a minimum in yield at mass-symmetry (left) to a narrow peak at symmetry, which shows no evidence of a mass-angle correlation.

ber of magic numbers in the entrance channel $\mathrm{N}_{\text {Magic }}$, and then by the difference $\Delta(\mathrm{N} / \mathrm{Z})$ between the $\mathrm{N} / \mathrm{Z}$ values of the target and projectile nuclei. The left-most reaction has only a single magic number in the entrance channel, and shows a U-shaped mass distribution, consistent with MAD1, as expected from systematics (right-hand purple circle in Fig.2). With two magic numbers, the reactions better matched in $\mathrm{N} / \mathrm{Z}$ (smaller values of $\Delta(\mathrm{N} / \mathrm{Z})$ ) show a peak at mass-symmetry, associated with an angleindependent ridge in the MAD. With three magic numbers, but less favourable $\Delta(\mathrm{N} / \mathrm{Z})$, a similar result is observed.

Fits were performed to the mass-ratio spectra, including an asymmetric U-shaped background from fast quasifission (derived from the reactions without a masssymmetric peak) and a mass-symmetric Gaussian peak. As the beam energy for the $\mathrm{Cr}+\mathrm{Pb}$ reactions is increased, Fig.6(a) shows that the mass-symmetric peak progressively becomes a smaller fraction of the total fission yield within the range $0.3<\mathrm{M}_{R}<0.7$. This might be expected, as the relative velocity at contact increases, as does the contribution from high angular momenta, for which the potential energy surface will be more repulsive. From the symmetric yield fraction, and preliminary absolute cross section normalization, estimates of the cross sections for the symmetric yield were extracted, as shown in Fig.6(b). Also shown are the xn evaporation residue cross sections
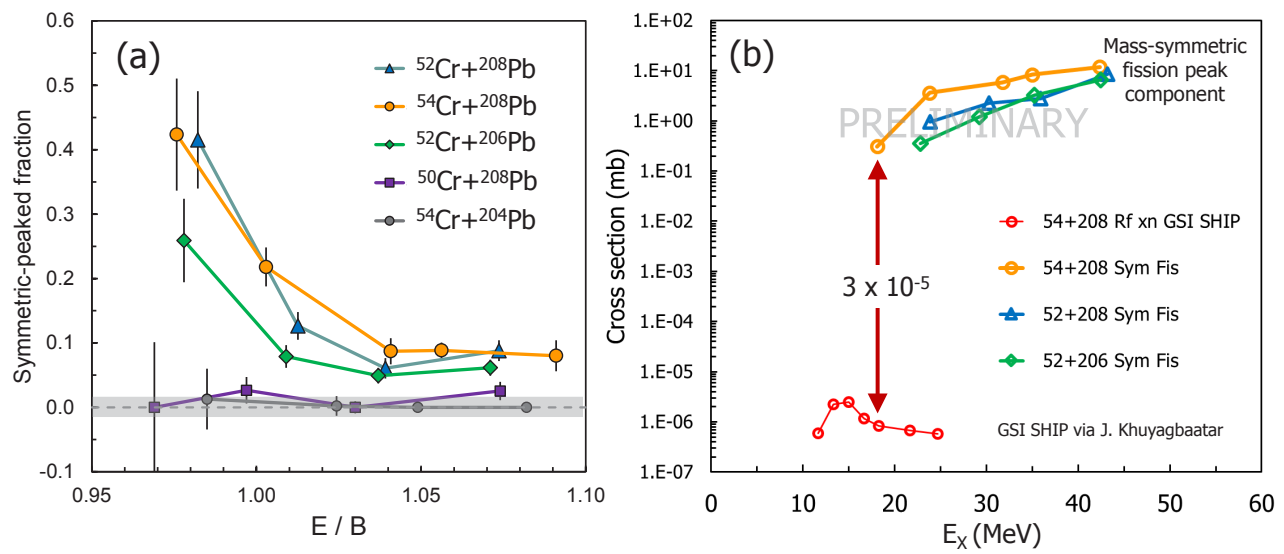

Figure 6. (a) The extracted fraction of symmetric peaked fission events to all fission and quasifission events in the range $0.3<\mathrm{M}_{R}<0.7$, for the indicated $\mathrm{Cr}+\mathrm{Pb}$ reactions, as a function of beam energy with respect to the capture barrier energy (E/B). The smooth fall with increasing E/B might be expected. (b) Preliminary cross sections for the symmetric-peaked fission component for each reaction (where present) as a function of compound nucleus excitation energy. The summed xn evaporation residue cross sections for the ${ }^{54} \mathrm{Cr}+{ }^{208} \mathrm{~Pb}$ reaction are also shown. 
for the ${ }^{54} \mathrm{Cr}+{ }^{208} \mathrm{~Pb}$ reaction [22]. If the symmetric yield originated only from fusion-fission, this would correspond to a probability of surviving fission decay of $3 \times 10^{-5}$ at $\sim 18 \mathrm{MeV}$ excitation energy. The suppression of SHE cross sections from fission competition in this reaction is much larger than from fast quasifission, though it is still not clear whether the mass-symmetric peak might have a contribution from slow quasifission.

These $\mathrm{Cr}+\mathrm{Pb}$ reactions show qualitatively similar changes in MAD as the ${ }^{40,44,48} \mathrm{Ca}+{ }^{204,208} \mathrm{~Pb}$ reactions. However, the transition from a U-shaped mass distribution to a narrow peak at mass-symmetry is an even more drastic change in reaction outcome, and indicates a definite bifurcation in reaction trajectories that is not so apparent in the $\mathrm{Ca}+\mathrm{Pb}$ MADs, since the quasifission yield is also shows a mass-symmetric peak. This is consistent with bifurcations in reaction trajectories between quasifission and fusion for a given projectile-target combination, evidenced by the observation of both quasifission and ER cross sections.

In reactions forming $\mathrm{Sg}(\mathrm{Z}=106)$, Fig. 2 indicates that a MAD of class 3 (no mass-angle correlation) would be expected only for $Z_{p} Z_{t}<1200$ (arrowed purple circle), in the absence of the favourable effects of magic numbers. This would correspond to a reaction with an Al projectile or lighter, with around half the atomic number of $\mathrm{Cr}$. The dramatic change in reaction outcome with a small change in neutron number in the $\mathrm{Cr}+\mathrm{Pb}$ reactions indicates that the observed systematic behaviour observed at higher beam energies does not necessarily allow prediction of behaviour in near-barrier reactions, where the nuclear structure of the system can play a very significant role. It is notable that this characteristic is also the case in comparing spontaneous and low energy nuclear fission with fission at high excitation energies.

\section{Effect of deformation alignment}

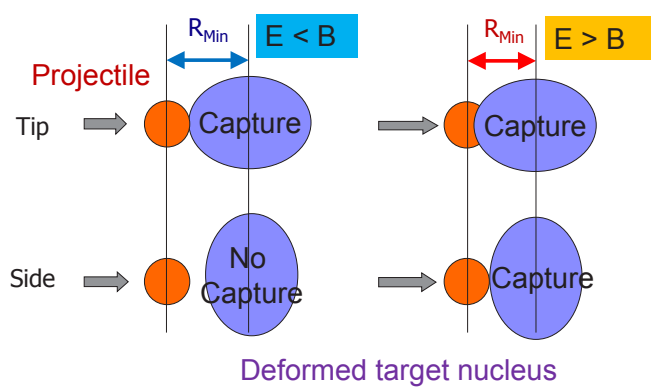

Figure 7. At beam energies below B, only deformation-aligned collisions result in contact and capture, whilst at $\mathrm{E} / \mathrm{B}>1$, the distance of closest approach $\left(\mathrm{R}_{\text {Min }}\right)$ is smaller (red arrow), thus capture also occurs for more compact equatorial configurations, giving longer sticking times, expected to be correlated with larger $\mathrm{P}_{C N}$.

Static deformation of the heavy reaction partner affects nuclear collisions because the capture barrier height depends on the relative orientation of the projectile nucleus and the deformation axis of the statically deformed heavy reaction partner [6]. For beam energies below the average barrier energy $B$, in collisions with prolate-deformed nuclei, it is only collisions in the "deformation aligned" configuration, giving axial or tip collisions (illustrated on the left in Fig.7) that result in capture. This is because the distance of closest approach at energies below the average barrier does not pass inside the smaller barrier radius corresponding to anti-aligned (side or equatorial) collisions. At energies well-above $B$, the distance of closest approach is reduced, and is inside the anti-aligned capture barrier. Thus, all orientations contribute to capture [7, 23].

An important question relevant to SHE synthesis is whether the elongated contact configuration (axial collision) or compact configuration (equatorial collision) affects the subsequent reaction dynamics. This has been most clearly demonstrated by the dependence on beam energy of the MAD characteristics [5, 10, 11, 24] and also mass-distributions $[6,25]$, for measurements from below to above the average capture barrier. Already in 1995, interpretation of measurements of fission angular distributions from below to above-barrier [7, 23, 26] led to the suggestion [23] that deformation alignment should be important in SHE synthesis. Specifically, as a result of the elongated contact configuration, the formation of a compact compound nucleus, and thus SHE cross sections, was suggested to be suppressed at $\mathrm{E} / \mathrm{B}<1$. Experimental evaporation residue cross sections $[27,28]$ have confirmed the correlation between fission characteristics and heavy element yields from below- to above-barrier. Indeed, superheavy element yields from reactions with prolate deformed actinide target nuclei have only been found at beam energies above predicted average capture barrier energies.

Let us now look at experimental results for collisions with prolate actinide nuclei. Fig.8 shows mass-angle distributions (with projected mass-ratio spectra below) for four different measurements. Panels (a) and (b) present the "reference" reaction ${ }^{48} \mathrm{Ca}+{ }^{208} \mathrm{~Pb}$ with $\mathrm{Z}_{p} \mathrm{Z}_{t}=1640$ at a subbarrier energy (already presented). This exhibits no massangle correlation and a narrow mass-width. As discussed previously, this is now believed to result from the magic numbers and the N/Z matching in the entrance channel. Fig.8(c) and (d) show data for the reaction ${ }^{34} \mathrm{~S}+{ }^{232} \mathrm{Th}$ [5], measured at a below-barrier energy. This reaction has a smaller $\mathrm{Z}_{p} \mathrm{Z}_{t}$ of 1440 , but has no magic numbers. Higher statistics in this measurement than obtained for the simi$\operatorname{lar}{ }^{34} \mathrm{~S}+{ }^{232} \mathrm{Th}$ reaction [11] show clearly that the dominant process is fast quasifission, with limited mass flow from the projectile/target mass partition, and less than $\sim 90^{\circ}$ of angular rotation. On the basis of $\mathrm{Z}_{p} \mathrm{Z}_{t}$ and $\mathrm{Z}_{C N}$, the systematic shown in Fig. 2 would predict a mass-angle correlation, but with symmetric peaked fission. Thus the deformation aligned collisions give MAD characteristics of a collision of heavier nuclei. The vertical dashed lines show the mass-ratio expected if one of the fragments was ${ }^{208} \mathrm{~Pb}$ the measured mass-splits closely follow these lines, a feature addressed in detail in Ref. [5]. There also appears to be a distinct component giving events peaked at masssymmetry, but with lower probability. This can almost certainly be associated with a component having a significantly longer sticking time. 

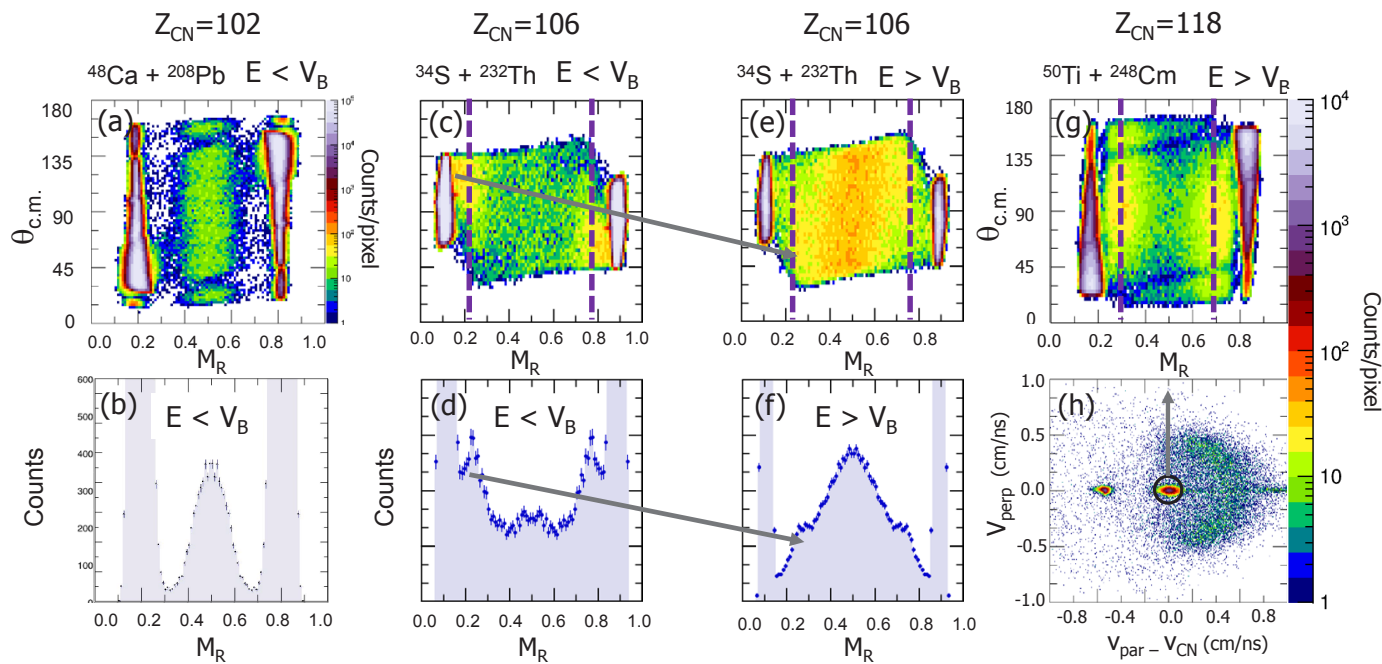

Figure 8. MAD and projected mass distributions for the four reactions indicated (see text). For the ${ }^{50} \mathrm{Ti}+{ }^{248} \mathrm{Cm}$ reaction, rather than the mass distribution, panel (h) shows the source velocity deduced assuming binary kinematics. The x-axis shows the component parallel to the beam (expressed as the difference between the measured velocity and the calculated centre-of-mass velocity), whilst the $y$-axis shows the component perpendicular both to the fission plane and the beam axis [7]. For a binary event originating from capture (corresponding to full momentum transfer from the projectile), the events should be close to the centre of the plot, around $(0,0)$. The group of events at $\left(-\mathrm{V}_{C N}, 0\right)$ correspond to spontaneous fission of the ${ }^{248} \mathrm{Cm}$ target. The MAD for ${ }^{50} \mathrm{Ti}+{ }^{248} \mathrm{Cm}$, gated as shown in (h), was measured at an energy above the expected barrier energy. It most closely resembles that for the ${ }^{34} \mathrm{~S}+{ }^{232} \mathrm{Th}$ reaction at the sub-barrier energy shown in (c).

Fig.8(e) and (f) show data for the same reaction at an above-barrier energy. The mass-asymmetric component now makes up a small fraction of the total fission-like events. Its mass-centroid is essentially unchanged [5], as indicated by the dashed lines, but the group has moved from backward to more forward angles (both characteristics indicated by the arrows). This can be associated with increased mean angular momentum at this abovebarrier energy, allowing a greater rotation angle (in a given time) before scission. The predominance of the group of events around mass-symmetry (but having a clear massangle correlation) is associated with the higher probability of equatorial collisions. The three dimensional geometry (resulting in a $\sin \theta$ probability weighting [7]) means that for prolate nuclei, the probability of equatorial collisions at energies well-above the average barrier energy is much higher than that of axial (deformation aligned) collisions. The mass-angle correlation shows, despite the compact contact configuration in equatorial collisions, that these collisions predominantly result in quasifission, in contrast with the sub-barrier ${ }^{48} \mathrm{Ca}+{ }^{208} \mathrm{~Pb}$ data, which shows no mass-angle correlation. Although equatorial collisions with a prolate deformed nucleus do seem to result in longer sticking times and increased $\mathrm{P}_{C N}$ compared with an equivalent spherical nucleus, the comparison of MADs (a) and (e) suggests that the enhancement is not as great as the effect of colliding two doubly-magic neutron-rich nuclei at a sub-barrier energy. More detailed analysis of a larger number of measurements is needed to reach a more quantitative conclusion about this factor in SHE formation reactions.

For all reactions with actinide target nuclei, there is a non-negligible probability of sequential fission of the target-like nucleus after the transfer of nucleons between the colliding nuclei early in the reaction. The analysis method allowing clean separation of the full momentum transfer fission following capture is illustrated for the ${ }^{50} \mathrm{Ti}+{ }^{248} \mathrm{Cm}$ reaction in Fig.8(h). The tightly grouped events in the centre of the graph $(0,0)$ correspond to binary events $[4,7]$, originating from a source with the velocity of the centre-of-mass in the collision, with only a small momentum spread due to neutron evaporation. The surrounding broad semi-circular distribution of events corresponds to three-body events, most likely of fission of various target-like nuclei resulting from nucleon transfers, with the projectile-like nuclei recoiling with various angles and energies giving a broad distribution of velocities to the target-like nuclei. The tight group of events at $\left(-\mathrm{V}_{C N}, 0\right)$ correspond to spontaneous fission of the ${ }^{248} \mathrm{Cm}$ target nuclei, stationary in the laboratory frame. These show the expected asymmetric-peaked fission mass distribution. A typical gate on FMT events, as used to generate the MAD in Fig. $8(\mathrm{~g})$, is shown by the black circle.

The ${ }^{50} \mathrm{Ti}+{ }^{248} \mathrm{Cm}$ reaction forms the compound nucleus ${ }^{298} \mathrm{Og}(\mathrm{Z}=118)$. The MAD shown in Fig.8(h) was measured at the Australian National University, using a target from Mainz/GSI, at a beam energy above the predicted [29] average capture barrier energy, where equatorial collisions having the longest sticking times would be expected to be dominant. However, the MAD more closely resembles the sub-barrier measurement for ${ }^{34} \mathrm{~S}+{ }^{232} \mathrm{Th}$ shown in Fig.8(c), rather than the above-barrier measurement shown in Fig.8(e). The relative yield of mass-symmetric events is smaller than for the sub-barrier ${ }^{34} \mathrm{~S}+{ }^{232} \mathrm{Th}$ reaction, but the fast quasifission events still show a considerable mass flow towards mass-symmetry. As in panels (c) and (e), the dashed lines show the ex- 
pected mass-ratio if one of the quasifission fragments were ${ }^{208} \mathrm{~Pb}$.

The role of ${ }^{208} \mathrm{~Pb}$ in quasifission mass distributions was investigated in the ${ }^{40} \mathrm{Ca}+{ }^{238} \mathrm{U}$ reaction, both experimentally and through TDHF calculations [15]. It was concluded that the closed shells centred on ${ }^{208} \mathrm{~Pb}$ play a strong role in determining the mass-splits, but for that reaction, only for axial (tip) collisions. In the ${ }^{50} \mathrm{Ti}+{ }^{248} \mathrm{Cm}$ reaction, the yield around ${ }^{208} \mathrm{~Pb}$ seems to be associated with all orientations. Various possible contributions to the observed behaviour need to be investigated. These include the possible effect of shells in the lighter fragment, sequential fission of heavy target-like nuclei, deviation of the orientation-dependent effective capture barriers from expectations, and changes in dynamics resulting from the heavier nuclei involved in the collision.

A program of measurements of quasifission (including MAD over a wide angular range) for projectiles from ${ }^{48} \mathrm{Ca}$ to ${ }^{64} \mathrm{Ni}$, bombarding targets from ${ }^{208} \mathrm{~Pb}$ to ${ }^{249} \mathrm{Cf}$, is in progress at the ANU. Analysis and interpretation of this body of data will give more quantitative insights into SHE synthesis reactions.

\section{Conclusions}

Mass-angle distributions give the most direct information on the characteristics and time scales of quasifission. A systematic study of carefully chosen mass-angle distributions has provided information on the global trends of quasifission. Large deviations from these systematics reveal the major role played by the nuclear structure of the two colliding nuclei in determining the quasifission probability and time scale, and thus implicitly in hindering or favouring superheavy element synthesis.

The observation of rapid changes in quasifission outcomes, depending on magicity, neutron number, and beam energy is a severe challenge for models of quasifission and SHE formation to reproduce. And yet this level of sensitivity of reaction dynamics to nuclear structure is what models must attempt to reproduce, in order to map out the optimum experimental opportunities to create new superheavy elements and isotopes in the future.

\section{References}

[1] D.J. Hinde, M. Dasgupta, A. Mukherjee, Phys. Rev. Lett. 89, 282701 (2002)

[2] J. Tōke, B. Bock, G.X. Dai, A. Gobbi, S. Gralla, K.D. Hildenbrand, J. Kuzminski, W. Müller, A. Olmi, H. Stelzer, Nucl. Phys. A 440, 327 (1985)

[3] R. du Rietz, D.J. Hinde, M. Dasgupta, R.G. Thomas, L.R. Gasques, M. Evers, N. Lobanov, A. Wakhle, Phys. Rev. Lett. 106, 052701 (2011)

[4] R. du Rietz, E. Williams, D.J. Hinde, M. Dasgupta, M. Evers, C.J. Lin, D.H. Luong, C. Simenel, A. Wakhle, Phys. Rev. C 88, 054618 (2013)

[5] E. Prasad, A. Wakhle, D.J. Hinde, E. Williams, M. Dasgupta, M. Evers, D.H. Luong, G. Mohanto, C. Simenel, K. Vo-Phuoc, Phys. Rev. C 93, 024607 (2016)
[6] M. Dasgupta, D.J. Hinde, N. Rowley, A.M. Stefanini, Annu. Rev. Nucl. Part. Sci. 48, 401 (1998)

[7] D.J. Hinde, M. Dasgupta, J.R. Leigh, J.C. Mein, C.R. Morton, J.O. Newton, H. Timmers, Phys. Rev. C 53, 1290 (1996)

[8] W.Q. Shen, J. Albinski, A. Gobbi, S. Gralla, K.D. Hildenbrand, N. Herrmann, J. Kuzminski, W.F.J. Müller, H. Stelzer, J. Tōke et al., Phys. Rev. C 36, 115 (1987)

[9] R.G. Thomas, D.J. Hinde, D. Duniec, F. Zenke, M. Dasgupta, M.L. Brown, M. Evers, L.R. Gasques, M.D. Rodriguez, A. Diaz-Torres, Phys. Rev. C 77, 034610 (2008)

[10] R. Rafiei, R.G. Thomas, D.J. Hinde, M. Dasgupta, C.R. Morton, L.R. Gasques, M.L. Brown, M.D. Rodriguez, Phys. Rev. C 77, 024606 (2008)

[11] D.J. Hinde, R. du Rietz, M. Dasgupta, R.G. Thomas, L.R. Gasques, Phys. Rev. Lett. 101, 092701 (2008)

[12] D.J. Hinde, R.G. Thomas, R. du Rietz, A. DiazTorres, M. Dasgupta, M.L. Brown, M. Evers, L.R. Gasques, R. Rafiei, M.D. Rodriguez, Phys. Rev. Lett. 100, 202701 (2008)

[13] C. Simenel, D.J. Hinde, R. du Rietz, M. Dasgupta, M. Evers, C.J. Lin, D.H. Luong, A. Wakhle, Phys. Lett. B 710, 607 (2012)

[14] E. Williams, D.J. Hinde, M. Dasgupta, R. du Rietz, I.P. Carter, M. Evers, D.H. Luong, S.D. McNeil, D.C. Rafferty, K. Ramachandran et al., Phys. Rev. C 88, 034611 (2013)

[15] A. Wakhle, C. Simenel, D.J. Hinde, M. Dasgupta, M. Evers, D.H. Luong, R. du Rietz, E. Williams, Phys. Rev. Lett. 113, 182502 (2014)

[16] Hinde, D.J., du Rietz, R., Williams, E., Simenel, C., Lin, C.J., Wakhle, A., Cook, K.J., Dasgupta, M., Evers, M., Luong, D.H., EPJ Web of Conferences 66, 03037 (2014)

[17] Hinde, D.J., Williams, E., du Rietz, R., Dasgupta, M., Wakhle, A., Simenel, C., Luong, D.H., Cook, K.J., EPJ Web of Conferences 86, 00015 (2015)

[18] Hinde, D.J., Williams, E., Mohanto, G., Simenel, C., Dasgupta, M., Wakhle, A., Carter, I.P., Cook, K.J., Jeung, D.Y., Luong, D.H. et al., EPJ Web of Conferences 117, 08006 (2016)

[19] Hinde, D. J., Williams, E., Mohanto, G., Simenel, C., Jeung, D. Y., Dasgupta, M., Prasad, E., Wakhle, A., Vo-Phuoc, K., Carter, I. P. et al., EPJ Web Conf. 123, 03005 (2016)

[20] Hinde, D.J., Dasgupta, M., Jeung, D.Y., Mohanto, G., Prasad, E., Simenel, C., Walshe, J., Wahkle, A., Williams, E., Carter, I.P. et al., EPJ Web Conf. 131, 04004 (2016)

[21] D.J. Hinde, M. Dasgupta, Phys. Lett. B 622, 23 (2005)

[22] S. Hofmann, Progress in Particle and Nuclear Physics 62, 337 (2009), heavy-Ion Collisions from the Coulomb Barrier to the Quark-Gluon Plasma

[23] D.J. Hinde, M. Dasgupta, J.R. Leigh, J.P. Lestone, J.C. Mein, C.R. Morton, J.O. Newton, H. Timmers, 
Phys. Rev. Lett. 74, 1295 (1995)

[24] D.J. Hinde, R. du Rietz, M. Dasgupta, R.G. Thomas, L.R. Gasques, Phys. Rev. Lett. 101, 092701 (2008)

[25] K. Nishio, H. Ikezoe, S. Mitsuoka, I. Nishinaka, Y. Nagame, Y. Watanabe, T. Ohtsuki, K. Hirose, S. Hofmann, Phys. Rev. C 77, 064607 (2008)

[26] J.C. Mein, D.J. Hinde, M. Dasgupta, J.R. Leigh, J.O. Newton, H. Timmers, Phys. Rev. C 55, R995 (1997)
[27] S. Mitsuoka, H. Ikezoe, K. Nishio, K. Satou, J. Lu, Phys. Rev. C 65, 054608 (2002)

[28] K. Nishio, H. Ikezoe, I. Nishinaka, S. Mitsuoka, K. Hirose, T. Ohtsuki, Y. Watanabe, Y. Aritomo, S. Hofmann, Phys. Rev. C 82, 044604 (2010)

[29] W.J. Swiatecki, K. Siwek-Wilczyńska, J. Wilczyński, International Journal of Modern Physics E Vol. 13, No 1, 261 (2004) 